\title{
The Influences of Inorganic Scintillator Optical Fiber Radiation Dosimeter in Some Conditions
}

\author{
Zhuang Qin1, Yaosheng Hu1, Yu Ma1, Wenhui Zhao1, Weimin Sun1, Tianci Xie1, \\ Chuntong Jiang', Daxin Zhang², Ziyin Chen ${ }^{2}$, Boran Wang ${ }^{3}$, He Tian ${ }^{4}$, Elfed Lewis ${ }^{5}$
}

\author{
${ }^{1}$ Key Lab of In-fiber Integrated Optics, Ministry Education of China, Harbin Engineering University, Harbin, China \\ ${ }^{2}$ Comprehensive Cancer Center, First Affiliated Hospital of Harbin Medical University, Harbin, China \\ ${ }^{3}$ Harbin YIAOMI Technology Development Co., Ltd, Harbin, China \\ ${ }^{4}$ College of Science, Northeast Forestry University, Harbin, China \\ ${ }^{5}$ Optical Fibre Sensors Research Centre, University of Limerick, Castletroy, Limerick, Ireland \\ Email: sunweimin@hrbeu.edu.cn
}

How to cite this paper: Qin, Z., Hu, Y.S., Ma, Y., Zhao, W.H., Sun, W.M., Xie, T.C., Jiang, C.T., Zhang, D.X., Chen, Z.Y., Wang, B.R., Tian, H. and Lewis, E. (2017) The Influences of Inorganic Scintillator Optical Fiber Radiation Dosimeter in Some Conditions. Optics and Photonics Journal, 7, 8185. https://doi.org/10.4236/opj.2017.78B011

Received: May 29, 2017

Accepted: August 7, 2017

Published: August 10, 2017

\begin{abstract}
In order to meet the increasing demands of modern radiotherapy, real time in-vivo dose measurement has recently attracted significant attention. A small, flexible optical fiber radiation dosimeter, with high signal-to-noise ratio (SNR) that employs inorganic scintillator materials is presented. In this paper, some properties are investigated under special conditions, such as saturation properties when the intensity of the X-Ray is increased and the influence of the temperature of the environment. These properties are important to practical considerations if the sensor is to be successfully deployed in-vivo.
\end{abstract}

\section{Keywords}

Optical Fiber Sensor, Radiation Dosimetry, Inorganic Scintillator Materials

\section{Introduction}

It is widely accepted that it is important to accurately deliver the dose during radiotherapy to adequately damage to the tumor cells whilst ideally causing no harm to the surrounding healthy tissue. The quality of the delivery of radiation therapy treatment depends on the ability to predict and measure the absorbed dose received by the whole volume being irradiated. As a "gold standard" instrument for Quality Assurance (QA purposes), Ionization chambers (ICs) are the instrument of choice for clinicians and radiotherapists. However, their relatively large size and the requirement for high voltage power supply make them unsuitable for in-vivo and internal use during patient treatment. In order to 
meet this requirement, a plastic optical fibre (POF) scintillation dosimeter which do not require pressure, or humidity corrections, with a simple "scintillator-fiber-Photomultiplier tube" (PMT) structure has previously been presented by Beddar et al. [1]. However, this dosimeter has a low light conversion efficiency, which means that it has reduced performance e.g. in terms of its Signal to Noise Ratio (SNR) particularly in the presence of external noise sources such as Cherenkov radiation and changes in the sensor response due to external temperature fluctuations. Therefore, many methods have been investigated to subtract the Cherenkov component [1] [2] [3], but until now no plastic scintillation dosimeter have actually been successfully applied in practice.

Alternatively, inorganic scintillator materials have shown great promise partly due to their relatively high light conversion efficiency. McCarthy et al., Qin et al. and O'Keeffe et al. designed a novel structure which embedded one kind of inorganic scintillator materials $\left(\mathrm{Gd}_{2} \mathrm{O}_{2} \mathrm{~S}: \mathrm{Tb}\right)$ into the core of the PMMA fibers [4] [5]. In these investigations, the contribution from Cherenkov radiation was lower than the background level of the noise. This dosimeter has exhibited good repeatability, excellent dose linearity ( $R^{2}$ of 0.9999$)$ as well as being isotropic with respect to the radial angular dependence of its signal. In this paper, the saturation characteristics when the intensity of the X-Ray are increased and the temperature response are investigated.

\section{Method}

The experimental set up and placement of the equipment used in this investigation is shown schematically in Figure 1 which details a plastic fiber-optic dosimeter submerged in a water equivalent tank in a radiotherapy bunker room. The plastic fiber-optic dosimeter relies on the conversion of the incident radiation dose to a measurable visible optical signal by fluorescence. According to this phenomenon, the radiation dose could be tested by measuring the intensity of the fluorescence.

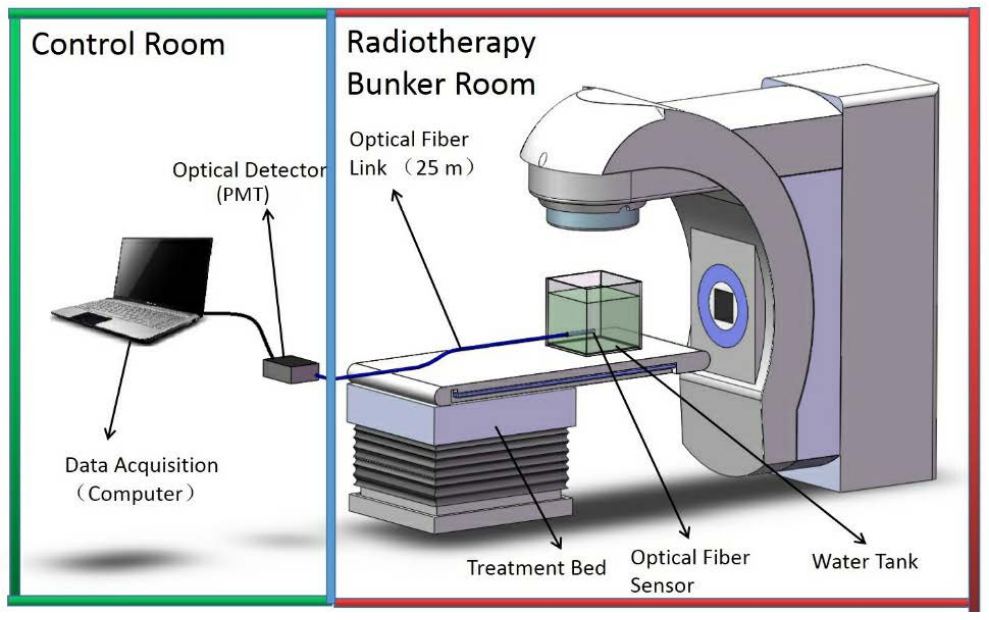

Figure 1. The device scheme of the experiment. 


\section{Result and Discussion}

\subsection{Saturation Property of the Inorganic Scintillator Materials}

Subject to fluorescence yield restrictions of the scintillation material, when the intensity of the X-ray increases, the inorganic scintillator materials must reach a saturation status. Experiments to investigate the saturation property of different scintillation materials were designed by increasing the dose rate in steps from $100 \mathrm{MU} / \mathrm{min}, 200 \mathrm{MU} / \mathrm{min}, 300 \mathrm{MU} / \mathrm{min}, 400 \mathrm{MU} / \mathrm{min}, 500 \mathrm{MU} / \mathrm{min}$ to 600 $\mathrm{MU} / \mathrm{min}$ (the maximum dose rate available from the clinical Linac of this investigation) at a submerged depth of $5 \mathrm{~cm}$ in water. Each exposure lasted 20 seconds.

Figure 2 shows the relationship between the intensity integrated over the full 20 seconds and the dose rate for two different scintilator materials. A linear regression analysis shows that the intensity of the dosimeter which use $\mathrm{Gd}_{2} \mathrm{O}_{2} \mathrm{~S}$ : $\mathrm{Tb}$ (Figure 2(a)) as the luminescent material followed a highly linear trend $\left(R^{2}\right.$ of over 0.9999). Therefore, within this range of dose rates it is clear that there exists no observable saturation. However, the results obtained using other scintillator materials e.g. $\mathrm{Gd}_{2} \mathrm{O}_{2} \mathrm{~S}$ :Pr exhibit a weaker linear trend (Figure 2(b)) due to the fact that the intensity of the fluorescence at $600 \mathrm{MU} / \mathrm{min}$ results in a sub-linear response. According to calculation, the actual intensity of $600 \mathrm{MU} / \mathrm{min}$ is only equivalent to the value when the dose rate is $564 \mathrm{MU} / \mathrm{min}$ had a perfectly linear trend been the case.

Similar experiments have been conducted using CsI: Tl, another scintillator material which also exhibits a limited saturation phenomenon, has been surveyed under different depth exposure conditions. In this case the dosimeter was placed at the depth of $5 \mathrm{~cm}$ and $10 \mathrm{~cm}$ in the water and the results of varying the dose rate between 100 and $600 \mathrm{MU} / \mathrm{min}$ in each case are shown in Figure 3.

Figure 3(a) shows that there is a clear saturation phenomenon at the depth of $5 \mathrm{~cm}$. According to calculation, the intensity of $600 \mathrm{MU} / \mathrm{min}(5.94 \mathrm{E}+06)$ is equivalent to the value at the dose rate is $579 \mathrm{MU} / \mathrm{min}$. But the result shows perfect linear trend ( $R^{2}$ of 0.9999 ) when tested at the depth of $10 \mathrm{~cm}$. The measured intensity $(5.37 \mathrm{E}+06)$ at $600 \mathrm{MU} / \mathrm{min}$ and a depth of $10 \mathrm{~cm}$ is less than the saturation point which occurs around an intensity value of $5.94 \mathrm{E}+06$ in the case of the $5 \mathrm{~cm}$ depth measurement.
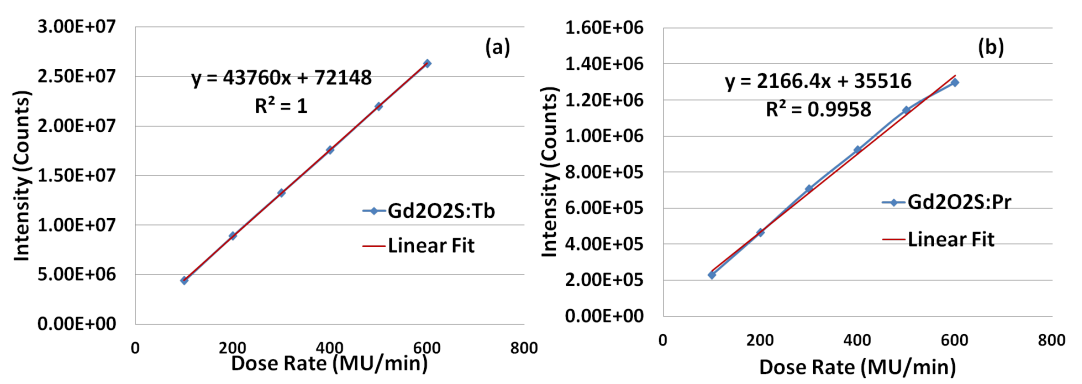

Figure 2. Intensity response at different dose rate for different Sensor materials (a) $\mathrm{Gd}_{2} \mathrm{O}_{2} \mathrm{~S}$ : Tb. (b) $\mathrm{Gd}_{2} \mathrm{O}_{2} \mathrm{~S}: \operatorname{Pr}$. 


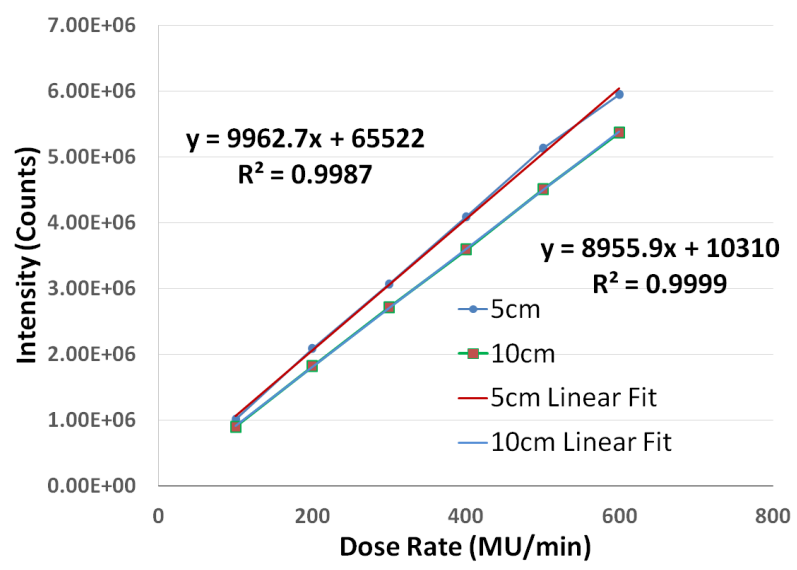

Figure 3. Intensity response at different depth in the water using CsI:Tl.

\subsection{Temperature Property of the Inorganic Scintillator Materials}

When the dosimeter is used in-vivo (within the human body), the temperature is about $37^{\circ} \mathrm{C}$, which is considerably higher than normal room temperature is $21^{\circ} \mathrm{C}$. It is therefore desirable to investigate the temperature response characteristics of the dosimeter. An experiment was conducted whereby the sensors was immersed in the water tank as shown in Figure 1 and the temperature was varied by regulating the temperature of the water in the tank to the desired value and recording the resulting output intensity. Four groups of experiments at different temperatures in the range $25^{\circ} \mathrm{C}$ to $40^{\circ} \mathrm{C}$ have been investigated using the dosimeter fabricated using the scintillator CsI:Tl. Figure 4 shows the results of varying the dose rate between 100 and $600 \mathrm{MU} / \mathrm{Min}$ and the temperature as stated above. Figure 4 clearly shows that the dosimeter is affected by the temperature, and that the intensity decreases linearly with temperature.

\section{Conclusion}

A series of experiments have been undertaken to establish the characteristics of inorganic scintillator materials when used for optical fiber radiation dosimetry under a wide range of operating conditions including dose rate, depth of immersion in water and temperature. The results indicate that inorganic scintillator materials show saturation phenomenon when the dose rate increase continuously. The saturation point of the materials that have been studied occur at a dose rate of over $550 \mathrm{MU} /$ with the exception of $\mathrm{Gd}_{2} \mathrm{O}_{2} \mathrm{~S}$ : $\mathrm{Tb}$ which exhibits no saturation effect across the full dose rate range tested. However, for normal treatment the dose rate is limited to $400 \mathrm{MU} / \mathrm{min}$ or less and in these circumstances the saturation effect would not occur. The output intensity of the dosimeter was also tested at different temperatures and it was found that the intensity varies with temperature linearly. Therefore, the temperature of the testing points must be known before using the inorganic scintillator materials dosimeter. However if this use is for internal dose measurement the temperature is relatively stable being about $37^{\circ} \mathrm{C}$ for humans. 


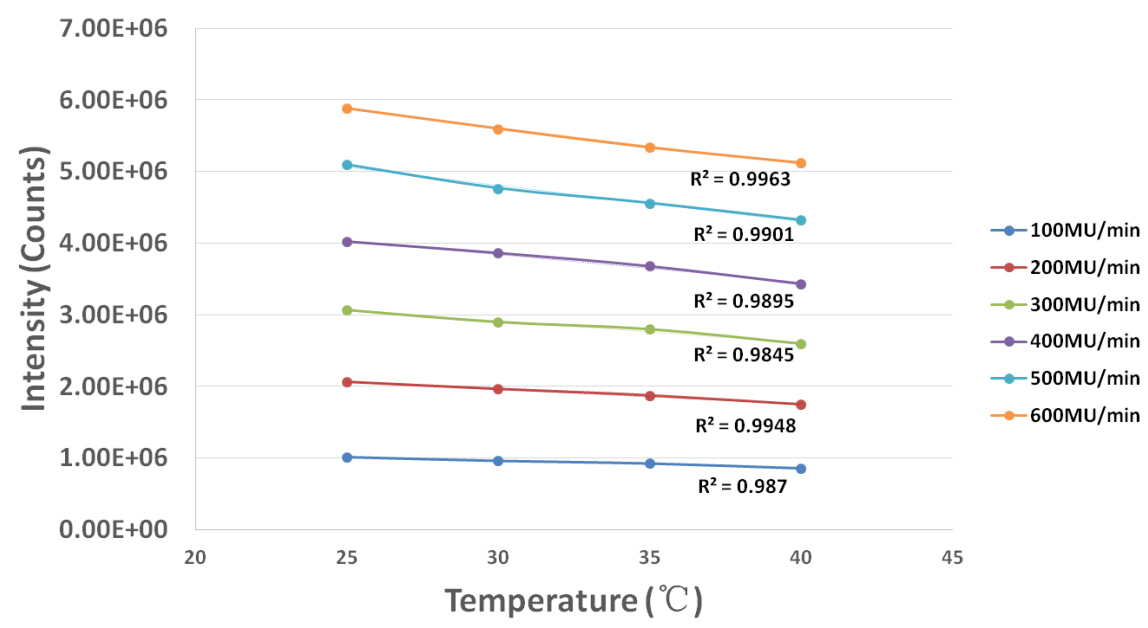

Figure 4. Intensity response at different temperature.

\section{Acknowledgements}

We are grateful for the assistance from Professor Zhang Hongquan and Huang Zongjun.

\section{References}

[1] Beddar, A.S., Mackie, T.R. and Attix, F.H. (1992) Water-Equivalent Plastic Scintillation Detectors for High-Energy Beam Dosimetry: I. Physical Characteristics and Theoretical Considerations. Phys. Med. Biol., 37, 1883-1900.

[2] Clift, M.A., Johnston, P.N. and Webb, D.V. (2002) A Temporal Method of Avoiding the Cerenkov Radiation Generated in Organic Scintillator Dosimeters by Pulsed Mega-Voltage Electron and Photon Beams. Phys. Med. Biol., 47, 1421-1433. https://doi.org/10.1088/0031-9155/47/8/313

[3] Boer, S.F., Beddar, A.S. and Rawlinson, J.A. (1993) Optical Filtering and Spectral Measurements of Radiation-Induced Light in Plastic Scintillation Dosimetry. Med. Phy., 38, 945-958. https://doi.org/10.1088/0031-9155/38/7/005

[4] MCarthy, D., O’Keeffe, S., Lewis, E., Sporea, D.G., Sporea, A., Tiseanu, I., Woulfe, P. and Cronin, J. (2014) Radiation Dosimeter Using an Extrinsic Fiber Optic Sensor. IEEE Sensors Journal, 14, 673-685. https://doi.org/10.1109/JSEN.2013.2284857

[5] Zhuang, Q., Yaosheng, H., Yu, M., Wenhui, Z., Weimin, S., Daxin, Z., et al. (2016) Embedded Structure Fiber-Optic Radiation Dosimeter for Radiotherapy Applications. Optics Express, 24, 5172-5185. https://doi.org/10.1364/OE.24.005172 
Submit or recommend next manuscript to SCIRP and we will provide best service for you:

Accepting pre-submission inquiries through Email, Facebook, LinkedIn, Twitter, etc. A wide selection of journals (inclusive of 9 subjects, more than 200 journals)

Providing 24-hour high-quality service

User-friendly online submission system

Fair and swift peer-review system

Efficient typesetting and proofreading procedure

Display of the result of downloads and visits, as well as the number of cited articles Maximum dissemination of your research work

Submit your manuscript at: http://papersubmission.scirp.org/

Or contactopj@scirp.org 